\title{
Multi - Agents Based Intelligent Information System For Virtual Manufacturing
}

Akela M. Al - Atroshi (PhD)

Professor
Abdulsatar M. Khudur (PhD)

Lecturer

\section{Sama Talee Azez Al - Aubaidy (PhD)}

Lecturer

\begin{abstract}
The current research concentrates on designing and applying an intelligent information system by the use of (Oracle). Distributed Database Management System based on multi - agents manufacturing process, in order to produce a new product by using the available potentialities of the company or by cooperating partners in the next future within the concept of the Virtual Manufacturing. Every Agent (user) has its own roles and privileges. The virtual manufacturing is a powerful production philosophy to provide a modeling and simulation to "manufacturing in the computer.

The research has special objectives in innovating a network among the Multi Agents in the Designed Proposed System and Planning priorities for Job Sequencing by the use of Genetic Algorithm logic. The product is designed in this agent by the use of the AutoCAD2004 software according to the specifications requested by the customer and the manufacturing capabilities of the company. Supervisor Agent has a vital and essential role in achieving compatibility and integration among the agents. It has the role of the DBA. The application results indicate that the VM philosophy has been performed efficiently by the use of the intelligent Multi - Agents which is managed and which attains integration by the server Agent that uses the available interfaces as Oracle DDBMS capabilities such as DDE, OLE and run - product. These capabilities have contributed in achieving quick response to the customers' orders.
\end{abstract}

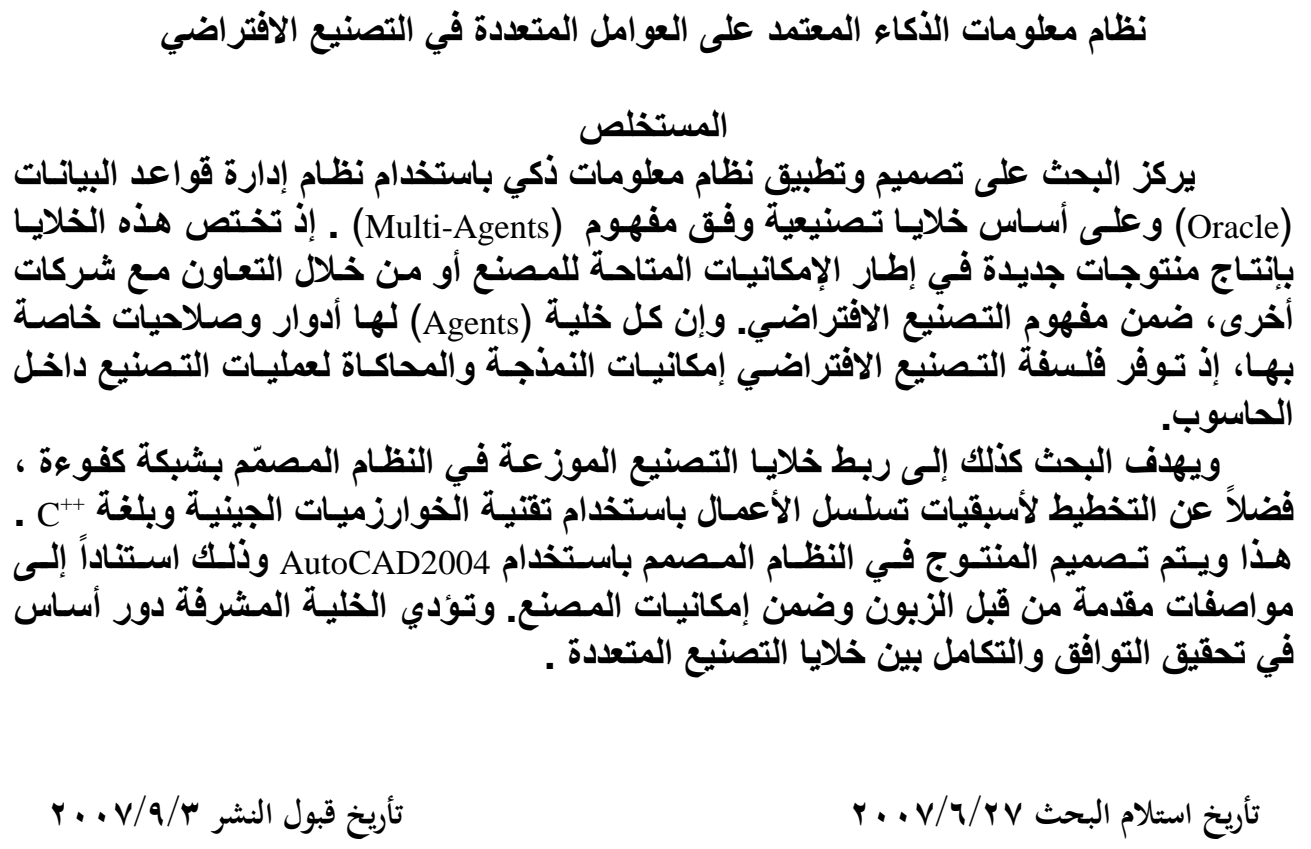




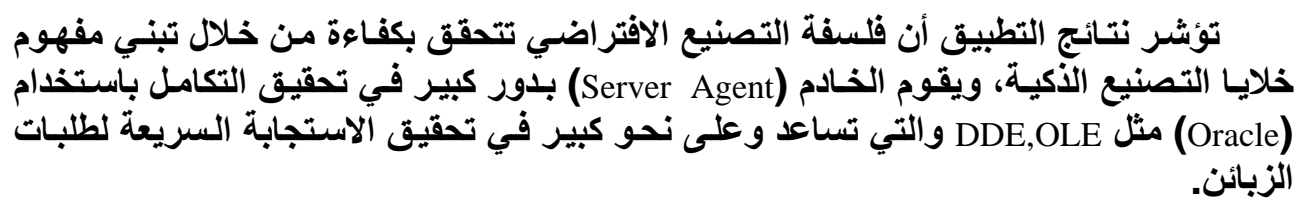

\section{Introduction to the Problem Domain}

The problem of this research has been derived from the role of information and communication technology in designing and applying the modern manufacturing philosophies. The problem includes the effect that the gap leaves between the Iraqi industrial companies and the huge development in the international manufacturing philosophies. Due to this effect, the industrial companies may face difficulties in survival, development and continuity in a highly competitive environment. The narrowing of the gap requires adopting one of the modern philosophies in manufacturing; so that these companies can develop to some extent. The basic and critical problem in the adoption decision is how to provide an intelligent information system that can support the planning operations and control the production of a product as well as providing certain mechanism for information exchange among production units within the company itself and other companies in remote geographical locations.

Our justification to choose the topic of this study which is "virtual manufacturing" lies in the field of Knowledge Engineering, and the Importance of the VM to the Iraqi Industry. It has been shown through the review of both theoretical and applied study of information and communication technology that there has been clear and insistent direction towards information systems and its intelligent application which is the cornerstone of virtual manufacturing in particular together with all manufacturing philosophies of the 21 st century. In changing environment, the enterprises are heavily focusing on cross- enterprise teams to share the intellectual capital in real time to produce the time to market in the product life cycle. This requires to involve suppliers, partners, customers and the knowledge workers with enterprise in the product life cycle processes.

According to what have been stated above, this study concentrates on designing and applying an intelligent information system based on manufacturing process to produce a new product by using the available potentialities of the company or by cooperating with partners in the future within the concept of the 'Agility'.

The research has the following special objectives:

Designing an intelligent information system by the use of (Oracle) language as a basis for the virtual manufacturing philosophy supporting the planning operations and controlling the product manufacturing within the computer's capabilities and its advanced technology.

Planning priorities for Job Sequencing by using of the Genetic Algorithm. 
Innovating a network among the manufacturing cells in the production system. This will be carried out according to the principle of the Multi Agents' concept within the framework of the supply chain.

Applying the designed system as a case study at the "National Company for Furniture Manufacturing in Mosul" at the level of producing an actual product in order to achieve an acceptable degree of compatibility between the designed system and the application environment.

2. The Mechanism of the Proposed System

The proposed system uses Agents based concurrent engineering application which is a system developed for the integration of design, manufacturing capability analysis, process planning and scheduling in framework of Virtual Manufacturing. To achieve the above mentioned system, an individual database for a local enterprise is being established which is then integrated via the Internet. [4].

Agile manufacturing makes use of modern information technology to form a virtual enterprise. Partners must maintain a high amount of communication and data exchange between themselves for success of the virtual enterprise. Data exchange does not mean just verbal communication; but rather the companies must exchange complete information about their products, all the way from design, manufacturing to inspection and shipping. [2] [7]

While, a virtual Enterprise is a temporary network of independent companies, suppliers, customers, design units and manufacturing process are linked by Information Technology (IT) to share skills, cost and access to one another. [1] [11]. The virtual manufacturing is a powerful production philosophy to provide a modeling and simulation to "manufacturing in the computer". [2] [13].

The proposed VM system represented by DB schema has been mainly designed by depending on 12 object types, 18 tables, 5 views, 1 trigger and 3 packages( $\left(^{*}\right)$. The package which includes a group of procedures or functions has been used to facilitate granting it an execution privilege by the users or agents to perform the tasks assigned to it. Figure (1) illustrates the work mechanism of the designed system according to DB schema. As regard to the roles and privileges of the proposed system, it is possible to say that the system has been designed on the basis of 5 agents. Every agent (user) has its own roles and privileges.

The supervisor has the role of Database Administrator (DBA) which entitles him all the roles and privileges available at the Oracle, because he is the General Manager. This also entitles him to give every agent roles and privileges according to the nature of their jobs. Accordingly, all the agents

() For more details, See Appendix (A); also see Appendix (B), (C),(D) for the details of how the structure of object type has been generated, in the thesis. 
can work cooperatively to perform the goal of the company efficiently and securely. To describe the information flow within a virtual manufacturing philosophy, we use a sample scenario to simulate actual ground and to illustrate its Operational Model which includes collaboration between an MRP planner in a high level planning and the design engineers in the virtual enterprise

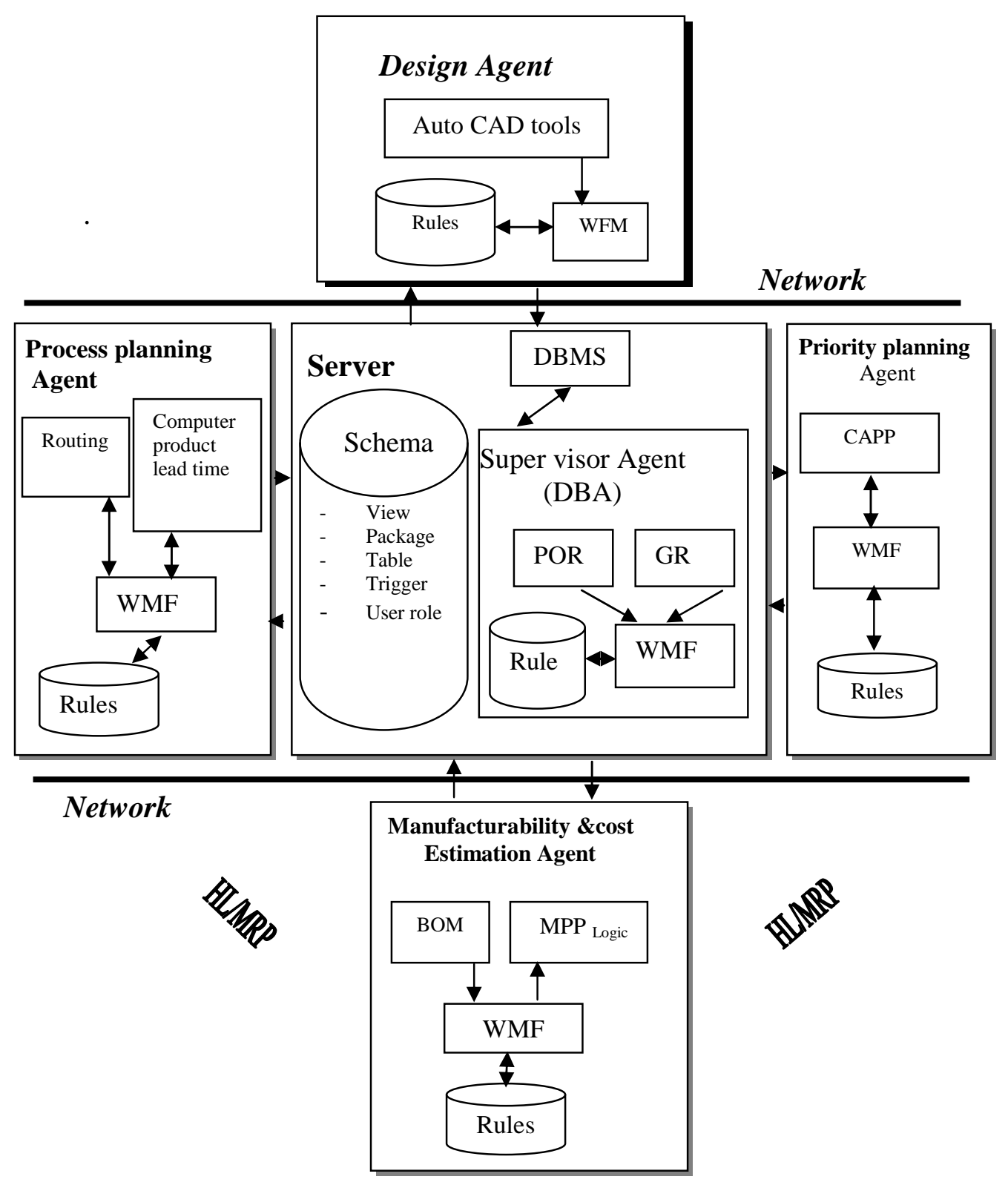

Figure 1

Mechanism of Proposed Information System. 


\subsection{Design Agent}

The Design Agent within the framework of the proposed system constitutes a very crucial stage for the manufacturing operation. The product is designed in this agent by the use of the AutoCAD2004 software according to the specifications requested by the customer and the manufacturing capabilities of the company. The Design Agent needs during the design processes information transfer among all the agents in the system through the communication network. As soon as the design is completed the design together with the attached data file are sent to the Related Agents by the process of the product manufacturing. Figure (2) illustrates the structure of the Design Agent. The Knowledge Processing Unit is distinguished to the expert rules by having a controlling information exchange. [9] [14]

The aim of CAD is to apply computers to both the modeling and communication of designs. Models of customer requirements for the design may develop and change as the design progresses, models of constraints on the design, imposed for example by available materials and manufacturing processes, these are the core of design process. CAD provides the designer with a variety of techniques for the definition of geometric entities (points, lines, arcs, conic section, and other curves). [5] [20]. A well designed CAD system should allow ease of repetition of detail, rapid modification of entities, modification and reuse of existing models, design the product simplicity and clarity, and help to reduce the number of parts in the product this reduce assembly costs and simplifies assembly. [3] [14].

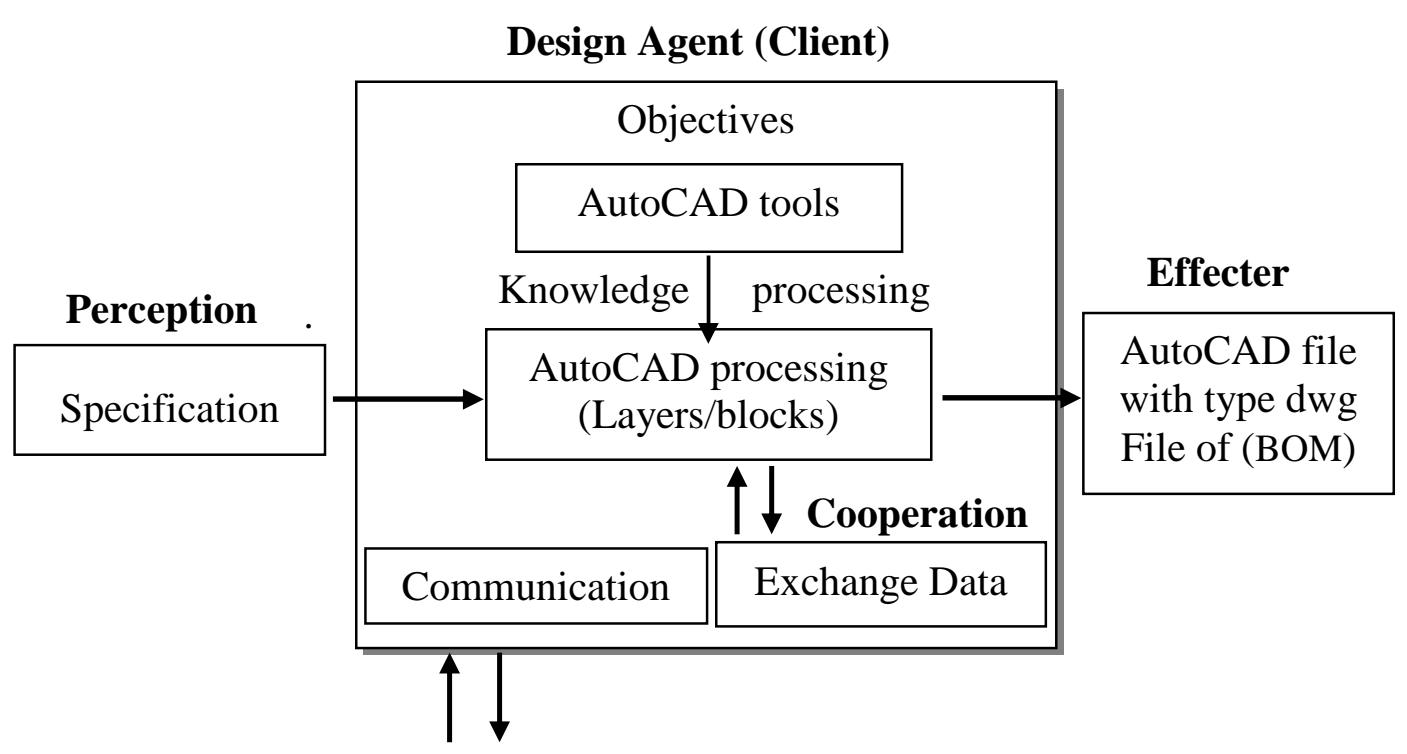

Figure 2

The Structure of the Design Agent. 
One application of a 3D model is in the generation of an engineering drawing by arranging multiple views of the model on a drawing sheet and then annotating these views with dimensions. The great advantage in 3D modeling when used to design complex modeled shapes for visualization or manufacture. The methods that have been developed for three dimensional modeling involve the representation of geometry as a collection of lines and other curves, or of surfaces, or of solids in space. The generation of realistic images involves the application of techniques in two distinct areas: the removal of hidden surfaces from the image, and the shading or coloring of the visible surfaces in a manner appropriate to the modeled lighting conditions. There are new techniques for representing the three dimensional modeling [3] [16]:

1. Wire Frame Geometry: In this the geometry is defined as a series (collection) of lines and curves representing the edges of, and perhaps sections through, the object. The name of this schema arises from the wire-like appearance of the models when viewed on a computer screen.

2. The Surface Representation Scheme: In which the component geometry is represented as a collection of surfaces, often attached to a wire frame. The most elementary of surface types is the flat plane, which may be defined in a number of ways including through a line and a point.

3. Solid Modeling: For many engineering purposes these representations are satisfactory. Any well-defined geometric property of any represented solid is to be calculated automatically. These models are like cuboids, cylinders, spheres, cones and the like.

In addition to entities, it can also use attributes (non geometric data) with the geometric model; these are typically name-value pairs and they are linked with design model elements through pointers from the elements to the attributes. The classic use of attributes (part name, part code, required quantities), is for the bills of materials from a computer based drawing or model (BOM derived from the attributes in the figure) [3]. However, the process of obtaining BOM, in this way, is still considered to be inefficient especially when it contains items that can not be designed like glue, paints, cotton and softening paper and the unfamiliarity of the designer with the codes of the items which are to be used in the database. Consequently, the form would be good enough to enter to the autocad software and to store BOM by selecting the required items from stock table. The SQL to view the $\mathrm{BOM}$ report so that the concerned agents can make use of it is as follows:

SELECT '( ' || p.prod_code $\|$ ' ) ' $\|$ p.prod.item.name1, b.mat_code, s.inventory.item.name1, s.inventory.item.name2, b.measure.qunt, b.measure.scale, s.loc_code

FROM bom $b$, product $\mathrm{p}$, stock $\mathrm{s}$

WHERE ((b.prod_code = :product_code) AND (p.prod_code = :product_code) AND (b.mat_code = s.mat_code)) 
ORDER BY s.loc_code $\|$ s.inventory.item.name1;

A CAD application environment is structured as a model with various layers. The partitioning of a drawing or model may be assisted by the use of layers or levels. Layers have two status, active layer being displayed and selectable, and inactive layer will not be displayed. The usefulness of layers depends on the extent to which the user is prepared to use them systematically to organize a model. On every office furniture design three layers have been generated. The first layer involves the final design for the product; the second layer shows the design of the product into individual parts showing how the parts can be constructed with its dimensions and the points of the holes (to fix the screws); the third layer explains the details [3] 14].

\subsection{Manufacturing Capability and Cost Estimation Agent(MC\&CE)}

This agent has a significant role by answering all the Design Agent enquiries as regard to technical available possibilities for manufacturing a new product and plans for its manufacturing. As soon as the design is completed a copy of the attached data file is sent to the MC\&CE agent. This file includes the technical equation of the product according to which the decision of the manufacturing process is to be taken and as follows:

1. If the raw materials and the parts are not generally available at the warehouse, the agent will issue a report of the required quantities with their measurement units to the supervisor agent who in his turn will send that report to the supplier in order to supply these requirements in time.

2. But if the manufacturing requirements are available, the agent will calculate the cost and price of each product included in the order separately then calculate the final price of the order and send a report to the supervisor agent who in his turn will send to the customer informing him of the prices.

The SQL which represents the stages of this agent work also represents as views of the manufacturing stages especially when comparing the stocked quantities with the actual required quantities for manufacturing the order is done. In addition to that, it includes the stage of calculating the costs and prices and as follows:

1. The stage of calculating the total quantity of the raw materials required for manufacturing the order. This stage is accomplished by stock_qunt_need_v because some of these materials enter also in a number of products in the customer's order.

CREATE
"VM_SYSTEM"."STOCK_QUNT_NEED_V"


FROM product_order $\mathrm{p}$, bom $\mathrm{b}$

WHERE p.prod_code $=$ b.prod_code

GROUP BY p.request.order_date, b.mat_code;

2. The stage of finding the latest date of the credit of each raw material entered in manufacturing the order: This stage is important to compare the current credit for the raw material in the stock with what the factory needs to manufacture the order.

CREATE OR REPLACE VIEW "VM_SYSTEM"

"BALANCE_ORDER_DATE_V" ("ORDER_DATE", "MAT_CODE",

"NEAREST_BALANCE_DATE") AS

SELECT p.request.order_date ,b.mat_code , $\max ($ s.stock.batch_date)

FROM product_order $\mathrm{p}$, bom $\mathrm{b}$, stock_balance $\mathrm{s}$

WHERE p.prod_code $=$ b.prod_code AND b.mat_code $=$ s.mat_code AND

s.stock.batch_date < p.request.order_date

GROUP BY p.request.order_date,b.mat_code;

3. The stage of mixing the credit dates with the total quantities of the material entered in manufacturing the order.

CREATE OR REPLACE VIEW "VM_SYSTEM". "NEED_NEAREST_BALANCE_V" ("ORDER_DATE", "MAT_CODE", "TOTAL_QUNT", "NEAREST_BALANCE_DATE") AS

SELECT s.order_date, s.mat_code, s.total_qunt, b.nearest_balance_date FROM stock_qunt_need_v s

LEFT OUTER JOIN balance_order_date_v b ON b.order_date $=$ s.order_date AND

ORDER BY s.order_date, s.mat_code;

b.mat_code $=$ s.mat_code

4. The stage of mixing the credit quantities and their prices with the total required quantities for manufacturing: This stage is done regardless of the unavailability of enough credit for the material entered in the order manufacturing.

CREATE OR REPLACE VIEW "VM_SYSTEM"."ITEM_ORDER_V" ("ORDER_DATE","MAT_CODE","TOTAL_QUNT","NEAREST_BA LANCE_DATE",

"QUNT_AVAILABLE","PRICE") AS

SELECT n.order_date, n.mat_code, n.total_qunt, n.nearest_balance_date, nvl(s.stock.qunt, 0$), \operatorname{nvl}($ s.stock.price, 0$)$

FROM need_nearest_balance_v n

LEFT OUTER JOIN stock_balance s ON

n.nearest_balance_date $=$ s.stock.batch_date AND

n.mat_code $=$ s.mat_code

ORDER BY n.order_date , n.mat_code; 


\subsection{Process Planning}

As soon as the this Agent receives the $\boldsymbol{B O M}$ file it will prepare itself to perform a number of activities upon receiving an informing from the Supervisor Agent:

1. It will define the routing of each new job in the order, otherwise it will search for the file and prepare it for use. The machines used for manufacturing each product are also defined.

2. It will also define the product's processing time with its parts and interior components as well as waiting periods for purchasing the orders. There is also setup-time for preparing machines for each manufacturing process. These have been taken into consideration when calculating the total time used for manufacturing the product, which is called the "lead time".

Calculating the lead time: The lead time means the total time spent in setup and operating every machine contributing in manufacturing the product, the SQL specialized in defining the routing and process time as follows:

CREATE OR REPLACE VIEW "VM_SYSTEM"."PROCESS_TIME_V" ("FACTORY","PROCESS_CODE","NAME1","NAME2","SETUP_TIME", "OPER_TIME") AS

SELECT P.factory, o.process_code, o.process.name1, o.process.name2, o.times.setup_time, o.times.oper_time

FROM operation_time o, prod_dept $\mathrm{p}$

WHERE p.mach_code $=\operatorname{trim}(\operatorname{substr}($ o.process_code $, 1,4))$;

But to calculate the lead time for a new product is as follows:

:prod_lead_time:=

technical_engineer_p.product_time(:path_tech.prod_code);

When any modification on the routing takes place a trigger calculate_lead_time is executed automatically to modify the lead time for a previously existing product and storing it respectively.

CREATE OR REPLACE TRIGGER "VM_SYSTEM"."CALCULATE_LEAD_TIME"

AFTER UPDATE OF "TIMES" ON "VM_SYSTEM"."OPERATION_TIME"

BEGIN

UPDATE product $\mathrm{p}$ SET p.prod.lead_time $=$

(SELECT sum(o.times.setup_time + o.times.oper_time)

FROM operation_time o, path_tech pt

WHERE o.process_code $=$ pt.process_code AND

pt.prod_code $=$ p.prod_code );

END;

\subsection{Priority Planning Agent (pp-Agent)}

Scheduling is defined as the problem of allocation of machines over time to complete jobs. The $\mathbf{m}^{*} \mathbf{n}$ job shop scheduling problem denotes a problem where a set of $\mathbf{n}$ jobs has to be processed on a set of $\mathbf{m}$ machines. 
Each job consists of a chain of operations, each of which requires a specified processing time on a specific machine [12][8][18]. The applied studies in this field have shown that the "Genetic Algorithm" is considered to be the most widely used technology in assigning machines jobs and obtaining best planning for jobs sequences on machines.

In case of performing sequence of jobs for the first time, they will be stored directly in the job_sched database table. If the optimal solution obtained is better than the stored one, it will be updated otherwise the database table will stay unchanged.

SELECT count(j.order_date) INTO c FROM job_sched j

WHERE j.order_date=:product_order.request_order_date AND f=j.fitness_fun; if $\mathrm{c}=0$ then

INSERT INTO job_sched

values(:product_order.request_order_date,p,f,spt,sft,sjl,act,utl,anj,ajl);

Else UPDATE job_sched SET

order_date $=:$ product_order.request_order_date, $j$ obs $=p$, fitness_fun $=f, \quad s \_p \_t=s p t$, $\mathrm{s} \_f \_t=s f t, s \_j \_l=s j l, a \_c \_t=a c t, u=u t l, a \_n \_j=a n j, a \_j \_l=a j l$

WHERE

(f=job_sched.fitness_fun

AND

:product_order.request_order_date=job_sched.order_date)

AND ( (job_sched.a_c_t $>$ act and $\mathrm{f}=$ 'average completion time') OR (job_sched.u $<$ utl and $\mathrm{f}=$ 'utilization') $\mathrm{OR}$ (job_sched.a_n_j $<$ anj AND $\mathrm{f}=$ 'average no. of jobs in the system')

OR

commit;

(job_sched.a_j_1 > ajl AND f='average job lateness') ); end if;

pl :=create_parameter_list('Q_1');

if not id_null(pl) then

add_parameter(pl,'d',text_parameter,to_char(:request_order_date,'DD-

MM-YY HH24:MI:SS'));

end if;

run_product(reports,'c:|reportsljob_seq.rdf',synchronous,runtime,filesystem, pl,null);

if not id_null(pl) then

end if;

destroy_parameter_list(pl);

Then the agent will issue a report about all the fitness functions that have been previously applied to the customer's order. Accordingly, the SQL report will be as follows:

SELECT js.order_date,js.jobs, js.fitness_fun, js.s_p_t, js.s_f_t, js.s_j_l, js.a_c_t, js.u, js.a_n_j, js.a_j_1

FROM job_sched js WHERE js.order_date = :d; 


\subsection{Supervisor Agent (SV-Agent)}

This agent has a vital and essential role in achieving compatibility and integration among the agents as it has the role of the DBA.

In case the manufacturer is not in a position that allows him to manufacture the order due to a shortage in the warehouse, this agent will inform the supplier with the short of raw materials with quantities and measurement unit.. This agent is also specialized in calculating the final price of the customer's order and calculating the total time required for treating the order and consequently deciding the delivery date of the customer's order.

This agent will perform the following procedures:

1. Distributing the customer's order to the concerned agents. The SQL report will be as follows:

SELECT po.request.order_date, p.prod.item.name1, po.request.qunt, po.request.due_date

FROM product p, product_order po

WHERE p.prod_code $=$ po.prod_code AND po.request.order_date $=:$ d;

Where $: \mathrm{d}$ represents the date of the order.

2. Informing the concerned warehouses with the decided issuing: Informing the concerned warehouses with issuing the quantities with their measurement unit to be delivered to the concerned factory to manufacture the order. Accordingly all the credits are modified automatically with the decided quantities to be issued. The SQL informing report will be as follows:

SELECT s.inventory.item.name1,i.qunt_available, i.total_qunt, i.qunt_available-i.total_qunt, s.inventory.mes_unt,sl.loc_name || ' ( || s.loc_code || ' )'

FROM item_order_v i, stock s, stock_loc sl

WHERE i.mat_code $=$ s.mat_code AND sl.loc_code $=$ s.loc_code AND i.order_date $=: \mathrm{d}$

ORDER BY sl.loc_name $\|$ s.inventory.item.name1;

3. Informing the customer with the cost of each product and the total cost of the whole order. The SQL report will be as follows:

SELECT pp.order_date, pp.prod_name, pp.work_cost, pp.manuf_cost, pp.mng_mrkt_cost,

pp.prod_cost, pp.profit, pp.prod_price, bc.item_name, bc.unit_price, bc.item_cost,

b.measure.qunt, b.measure.scale

FROM product_price_v pp, bom_cost_v bc, bom b, product p, stock s

WHERE ( (pp.order_date $=$ bc.order_date) AND (pp.order_date $=: \mathrm{d})$ AND AND

$\left(\right.$ pp.prod_name $=$ bc.prod_name) AND $\left(p p \cdot p r o d \_n a m e=p \cdot p r o d . i t e m . n a m e 1\right)$

(p.prod_code=b.prod_code) AND (bc.item_name= s.inventory.item. name1) AND 
(s.mat_code=b.mat_code) )

ORDER BY bc.item_name;

4. Calculating the official price of the order:

Order_price $=\sum_{i=1}^{N}$ prod $\_$price $(i) \times$ prod $\_$qunt $\_$need $(i)$.(11)
where $\mathrm{N}:$ No. of products in the order.

5. Calculating the order's delivery date:

Order_lead_time (Minutes) $=$

$\sum_{i=1}^{N}$ lead_time $(i) \times$ prod_qunt_need $(i)$

where $N$ : No. of products in the order.

The SQL of the special report for the last two procedures is as follows:

SELECT pp.prod_name, po.request.qunt, p.prod.lead_time, p.prod.lead_time * po.request.qunt Total_Lead_time(Minutes), po.request.due_date,

ceil (p.prod.lead_time $*$ po.request.qunt/360) Lead_Time(Days), pp.prod_price,

pp.prod_price * po.request.qunt Total_Price

FROM product_price_v pp, product $\mathrm{p}$, product_order po

WHERE pp.prod_name $=$ p.prod.item.name1 AND pp.order_date = po.request.order_date

AND p.prod_code $=$ po.prod_code AND pp.order_date $=: \mathrm{d}$

ORDER BY pp.prod_name;

\section{Interfaces between Agents}

Programming interface applications communicate with the database server by using a programming interface such as ODBC, JDBC, OLE, DDE, or embedded SQL. Each programming interface provides a library of function calls for communicating with the database. The interface library is located on each client computer (agent).

The open system architecture is supported by multiple interfaces and integration capacities (ActiveX, CAD, Oracle SQL, ODBC, XML, Socket, etc.) [17][19]. The Object Linking and Embedding (OLE) is a standard method, defined by Microsoft, for exchanging information between windows applications. Host applications (known as containers) are linked to OLE objects when they contain references to those objects. An embedded object, by contrast, contains an actual copy of the object [15][6]. OLE, is one of software tools, solutions and technologies that include the simulations tools, has been used for Process Control (OPC). OPC is an open interoperability industrial standard for sharing manufacturing information in an enterprise-wide manner. It is based on the Microsoft technologies of OLE. It provides 'plug-and-play' connectivity and interoperability between disparate automation devices, systems and software [10]. Forms Developer applications can be readily integrated with OLE such as spreadsheets, 
autocad, and word processors. The OLE built-in package allows your programs to access any functions or properties exposed by the object model of the OLE container. The DDE Package provides Dynamic Data Exchange (DDE) support within Developer components. DDE is a mechanism by which applications can communicate and exchange data in Windows. The PL/SQL package for DDE support provides application developers with an Application Programming Interface (API) for accessing DDE functionality from within PL/SQL procedures and triggers. The DDE functions enable Oracle applications to communicate with other DDE-compliant Windows applications (servers) in three ways: importing data, exporting data, and executing commands against the DDE Server.

The job of activating embodies the mechanism of the designed system as the nature of the work inside the manufacturing system implies integration and compatibility among the production units represented by the agents. Accordingly, the designs translate these relations into messages for exchanging information through the communication network among the agents and by the server. The figure (1) shows the flow of information between the agents and server through many modules which constitute the structure of the designed system. The Information system designed has the following features:

1. Local primitive information storage stores in server. DBMS manages local primitive information accessing, updating and constraint maintenance.

2. Application programs in each Agent (Client) access information storage through the WorkFlow Manager (WFM), and knowledge base stores agent policies and inter-agent protocols, support agent transaction workflow.

3. The agents (Clients and Server) are responsible for connecting to the network and transmitting information.

4. The workflow manager is responsible for converting agent queries to agent workflow. The workflow is defined as a collection of sub queries organized to fulfill a business task.

The messages sent between agents are either data text files (.dat), autocad files (.dwg) or reports files (.rdf). The agent writes these reports by the oracle report developer who has the capacity of either printing or sending the reports to the rest of the agents through LAN or the Internet as .htm, .pdf, XML files or through the E-mail. The most used interfaces for carrying these messages are explained below according to the nature of the agent.

\section{Implementation of Proposed System}

The system simulation involves selecting order from a number of orders received by the company during the period of the designed system 
application. The selected order are distinguished by having a number of jobs as explained in Table (1). These two orders have been selected due to the following reasons:

1.Selecting and applying planning jobs priorities software according to the genetic algorithm which demands the availability of several jobs to obtain efficient results.

2. The first order is distinguished by the fact that all the requirements for manufacturing all its jobs are available at the company's warehouses. On the other hand, the requirements needed for manufacturing the second order's jobs are unavailable and need to be provided by the suppliers.

Table (1) illustrates that the first order includes 7 jobs arriving at 9:0:0 on 10 July 2005 whereas the second order includes 3 jobs arriving at 10:0:0 on 1 October 2005. A product made of Melamen desk and a product made of a teak desk have been selected to follow up the manufacturing processes within the integration framework between the manufacturing stages represented by the Multi-Agents mentioned in the designed system.

Table 1

\section{Customer Order Report}

Customer_Order

\begin{tabular}{|c|c|c|}
\hline Jobs & Ount & Dusenato \\
\hline 3 Drawer Teak Desk $150 * 80 \mathrm{Cm}$ & 2 & 2 \\
\hline 4 Drawer Teak Desk $150 * 80 \mathrm{Cm}$ & 4 & 3 \\
\hline Warkaa Melamen Desk $1.50 * 80 \mathrm{Cm}$ & 3 & 3 \\
\hline Warkaa Melamen Desk Appendix $120^{*} 40 \mathrm{Cm}$ & 2 & I \\
\hline Ashor Tilipse Teak Desk $240^{\star} 120 \mathrm{Cm}$ & 3 & 2 \\
\hline Ashor Lillipse Melamen Desk $240^{*} 120 \mathrm{Cm}$ & 2 & 2 \\
\hline Ashor leak Desk Appendix $120 \% 40 \mathrm{Cm}$ & 3 & 2 \\
\hline 01-0CT-05 10:00:00 & & \\
\hline Joks & Qunt & Due Date \\
\hline Warkaa Melamen Desk Appendix $120 * 40$ Cm & 3 & 4 \\
\hline Warkaa Melamen Desk $150^{\star} 80 \mathrm{Cm}$ & 5 & 3 \\
\hline 4 Drawer Teak Desk $150^{\star} \overline{50} \mathrm{Cm}$ & 5 & 3 \\
\hline
\end{tabular}

\subsection{Product Design}

One of the main tasks of this agent after receiving the technical (engineering) specifications of the products, which reflects the customer's desire and the company's consent, is to start designing the prototype by the use of AutoCAD2004.

The product's designing stage, in the designed system, is distinguished by viewing the design in three forms. Each form is stored in a layer. Accordingly, each design file contains three layers. Figure (3) represents an unconnected structure for Melamen desk product and its appendix; the figure shows the product's measurement and dimensions. It also gives 
minute details on how to assemble the parts of the product i.e. it identifies the hole's cylinder dimensions used in assembling the interior components of the product.

Table (1) signifies the importance of the designing stage and the significant role of the information resulted from the design in creating the technical structure of the product (BOM) as well as achieving synchronization in manufacturing process and stages among the MultiAgents.

Figure 3

\section{Design Stages}
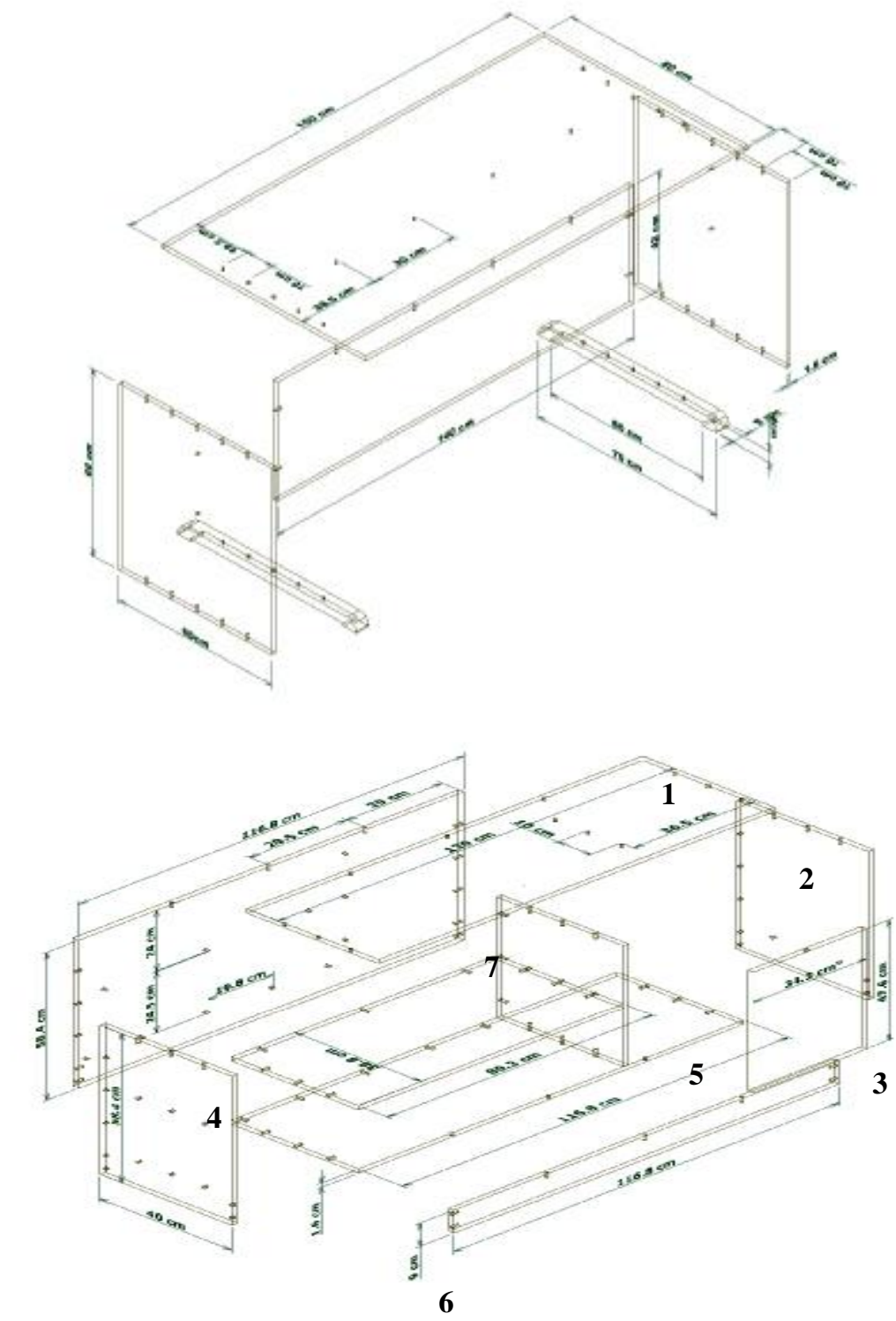
Table (1): Warkaa Melamen Desk (3-214).

Dimensions (Cm.): Height: 70, Width: 80, Length: 150.

Melamen Wood Dimensions (Cm.): $244 \times 122 \times 1.6$.

Oak Wood Dimensions (Cm.): $200 \times 10 \times 5$.

\begin{tabular}{|l|l|l|l|}
\hline Part No. & Parts of Desk & Parts Dimension $(\mathbf{C m})$. & Melamen Qty. \\
\hline 1 & Desktop & $150 \times 80$ & 1 \\
\hline 2 & The side & $66 \times 60$ & 2 \\
\hline 3 & Front Joint & $140 \times 42$ & 1 \\
\hline 4 & Legs & $70 \times 4$ & Oak Wood Qty. 2 \\
\hline
\end{tabular}

Table (2): Information Associated Product Design.

Dimensions (Cm.): Height: 60, Width: 40, Length: 120.

Melamen Wood Dimensions (Cm.): $244 \times 122 \times 1.6$.

\begin{tabular}{|c|c|c|c|}
\hline Part No. & Parts of Desk & Parts Dimension $(\mathbf{C m})$. & Melamen Qty. \\
\hline 1 & Desktop & $120 \times 40$ & 1 \\
\hline 2 & Sides & $58.4 \times 40$ & 2 \\
\hline 3 & Door & $47.6 \times 34.3$ & 1 \\
\hline 4 & Shelf & $80.3 \times 38.8$ & 1 \\
\hline 5 & Base & $116.8 \times 38.8$ & 1 \\
\hline 6 & Plate & $116.8 \times 9$ & 1 \\
\hline 7 & Middle side & $47.8 \times 38.4$ & 1 \\
\hline 8 & Back & $116.8 \times 58.4$ & 1 \\
\hline
\end{tabular}

\subsection{Manufacturability Evaluation}

The ME Agent receives the orders and then approves them through a constant and genuine dialogue with the customer. Relying on the attached file of the design, the ME Agent, according to the logic of HL/MRP treatment will:

1.Define the floors factory, which is the location of manufacturing the two products in the order, this will be done in agreement with the Design-

Agent.

2.Create a technical structure file for all the products' technical structures .

Table (3) represents the technical structure of the melamen desk with its appendix as well as the teak desk for each unit of the product. A copy of the $B O M$ file is then sent to the Supervisor Agent which in its turn sends it to the Related Agents. 


\subsection{Product Cost Estimation}

The MC\&CE-Agent receives a copy of BOM file for each product planned to be manufactured. The MC\&CE Agent will calculate the product's cost as soon as it receives an informing from the SV-Agent.

According to the logic of the MRP system treatment the total quantities of materials, parts and components needed for manufacturing each product are compared with the available credits at the company's warehouses. According to these procedures the following decisions will be taken:

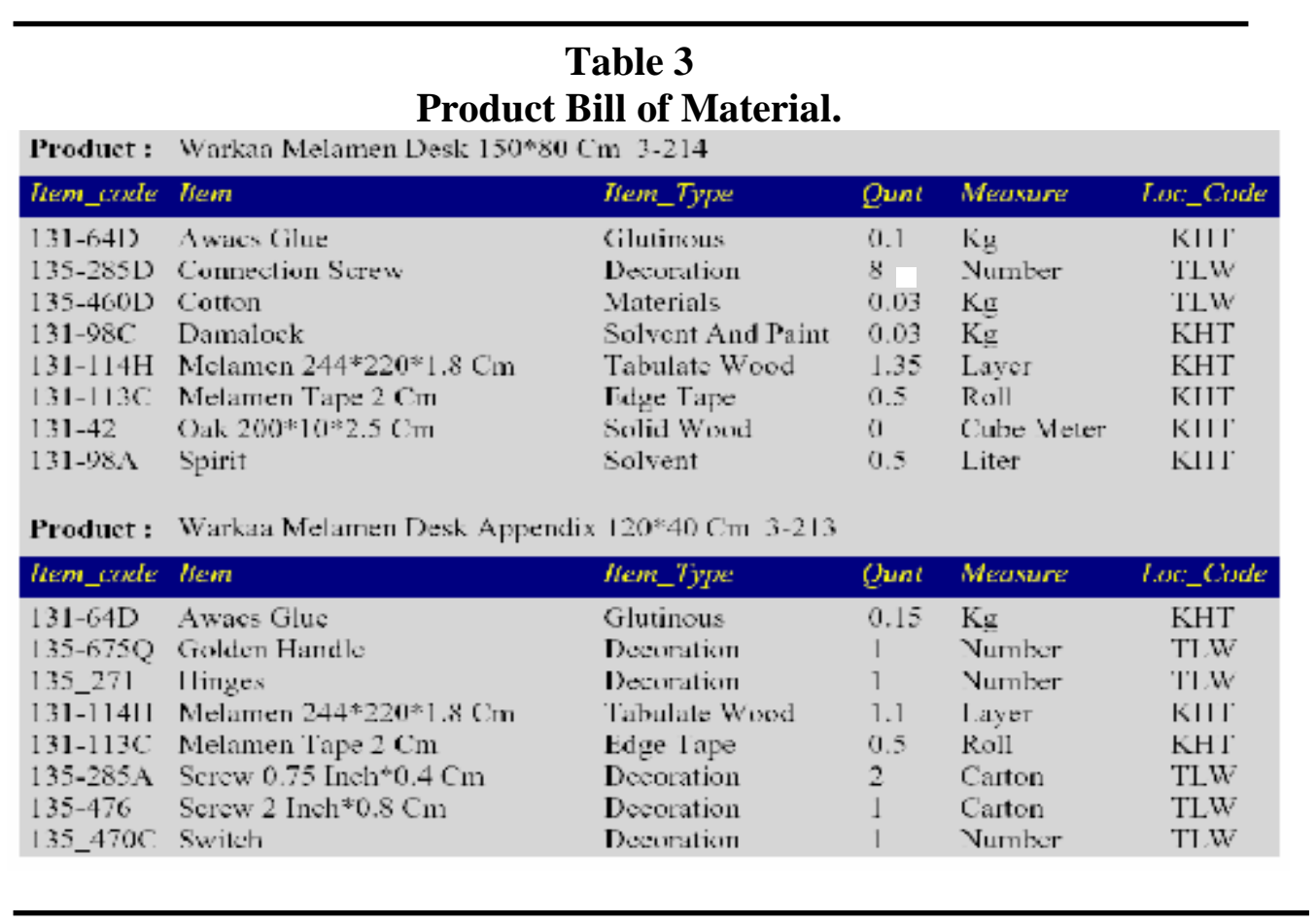

1. It has been confirmed by comparison that all the materials required for manufacturing all the jobs in the first order dated 9:0:0 10 July 2005 were available at the company's warehouses. The required quantities will be issued in due time.

2. It has been also confirmed that the materials required for the second order dated 10:0:0 1 October 2005 were not available at the company's warehouses. In this case a report of the required materials and quantities has to be prepared and sent to the supplier in order to be provided in the due time as explained in table(4). 


\section{Table 4}

Report Send to Supplier.

$$
\begin{aligned}
& \text { Request_Planning } \\
& \text { 01-0CT-05 10:00:00 }
\end{aligned}
$$

\begin{tabular}{|c|c|c|c|}
\hline Item Code & Item & Quantity Needed & Measure Unit \\
\hline $131-54$ & Bankal Liver $122 \div 244^{*} 0.4 \mathrm{Cm}$ & 2.3 & Layer \\
\hline $131-98 \mathrm{C}$ & Damalock & 1.125 & $\mathrm{Kg}$ \\
\hline $131+69 \Gamma$ & Dough & .125 & $\mathrm{Kg}$ \\
\hline $135-285 \Lambda$ & Screw 0.75 hach $0.4 \mathrm{~cm}$ & 6 & Carton \\
\hline $1.35-476$ & Serew 2 Inch ${ }^{8} 0.8 \mathrm{Cm}$ & 3 & Carton \\
\hline $131-98 \Lambda$ & Spiril & 7.5 & Liler \\
\hline $135+70 \mathrm{C}$ & Switch & 3 & Number \\
\hline $1.31-79$ & Teak 244 & 5.1 & Tayer \\
\hline
\end{tabular}

Table (5) shows the manufacturing requirements for all the jobs stated in the first order to be later issued from the company's warehouses.

\section{Table 5}

Manufacturing Requirements for the Order

Release_Order

\begin{tabular}{|c|c|c|c|c|}
\hline ftems & Item Available & Pull Quantity & Item Balance & Measure Unit \\
\hline Vail $25 \mathrm{~mm}$ & 30 & 19.5 & 10.5 & Carkm \\
\hline \multicolumn{5}{|c|}{ Stock: Materials Storage (135)/(TI W) } \\
\hline Ttems & Them_Available & Pull_Quantity & Them_Balance & Weasure_Unit \\
\hline Connection Serew & 768 & 44 & 724 & Numbar \\
\hline Cotton & 20 & 1.125 & 18.875 & $\mathrm{Kg}$ \\
\hline Goulden ITande & 100 & 37 & 6.3 & Number \\
\hline Hinges & 10 & 10 & a & Numbar \\
\hline Serew $0.751 \mathrm{nch}^{*} \mathrm{0} .4 \mathrm{Cm}$ & 10 & 9.5 & .5 & Carton \\
\hline Surew 2 lnah:0.8 $\mathrm{Cm}$ & 20 & 2 & 18 & Cirion \\
\hline Swikh & 30 & 14 & 16 & Numbsr \\
\hline \multicolumn{5}{|c|}{ Stock: Primary Items Storage $(131) ;(\mathrm{KHI})$} \\
\hline Frems & Ttem_Available & Pull_Quantity & Ttem_Ralance & Weasure_Unit \\
\hline Awats Glue & 200 & 15 & 185 & $\mathrm{~K}_{\mathrm{t}}$ \\
\hline Banal I ayor $122 * 244 * 0.4 \mathrm{Cm}$ & 270 & 8.826 & $26,1.174$ & Tayer \\
\hline Block Bard $\left.122^{\circ} 214^{*}\right] .8 \mathrm{~cm}$ & 30 & 15.122 & 14.878 & Layer \\
\hline Damaluek & 2.055 & 2.055 & 0 & $\mathrm{Kg}$ \\
\hline Dough & .15 & .15 & 0 & Kĭ \\
\hline Vahogany 201$)^{*} 10 \% 2.56 \mathrm{~m}$ & 31) & 012 & 29.98 & Gibe Veter \\
\hline Melamen $244^{\circ} 220^{*} 1.8 \mathrm{~cm}$ & 50 & 13.25 & 36.75 & Layer \\
\hline Velamen Tape $2 \mathrm{Cm}$ & 50 & 4.5 & 45.5 & Roll \\
\hline Oak $200 * 10^{*} 2.5 \mathrm{Cm}$ & 2666.80 & .4444 & $26 \times 1.4456$ & Cube Veter \\
\hline Spirit & 20 & 20 & 0 & Liter \\
\hline l'esk $244^{*} 122^{*}: 0.4 \mathrm{Cm}$ & 185 & 18.06 & 166.94 & Layer \\
\hline
\end{tabular}


According to the above mentioned information as regard to the costs; the costs of the planned products to be manufactured as well as the labor costs and manufacturing managerial and marketing expenses together with the profit margin will be calculated see table (6) for details.

\subsection{Priority Dispatching Rules}

The Priority Planning Agent receives the order.dat file from the process Planning Agent. This file contains the required information about the orders and is considered as one of the main inputs for those software designed by the use of $\mathrm{C}++$ language according to the genetic algorithm logic for planning job priorities in the shop floor.

By cooperation with the Supervisor Agent one of the rules relied on in designing software to plan for job priorities, is selected. The rule of the Average Completion time has been selected to be a fitness function according to which the optimal sequence for executing the manufacturing planned jobs has also been selected. This rule aims at reducing the lead time for products' manufacturing and achieving quick response for the customers' orders.

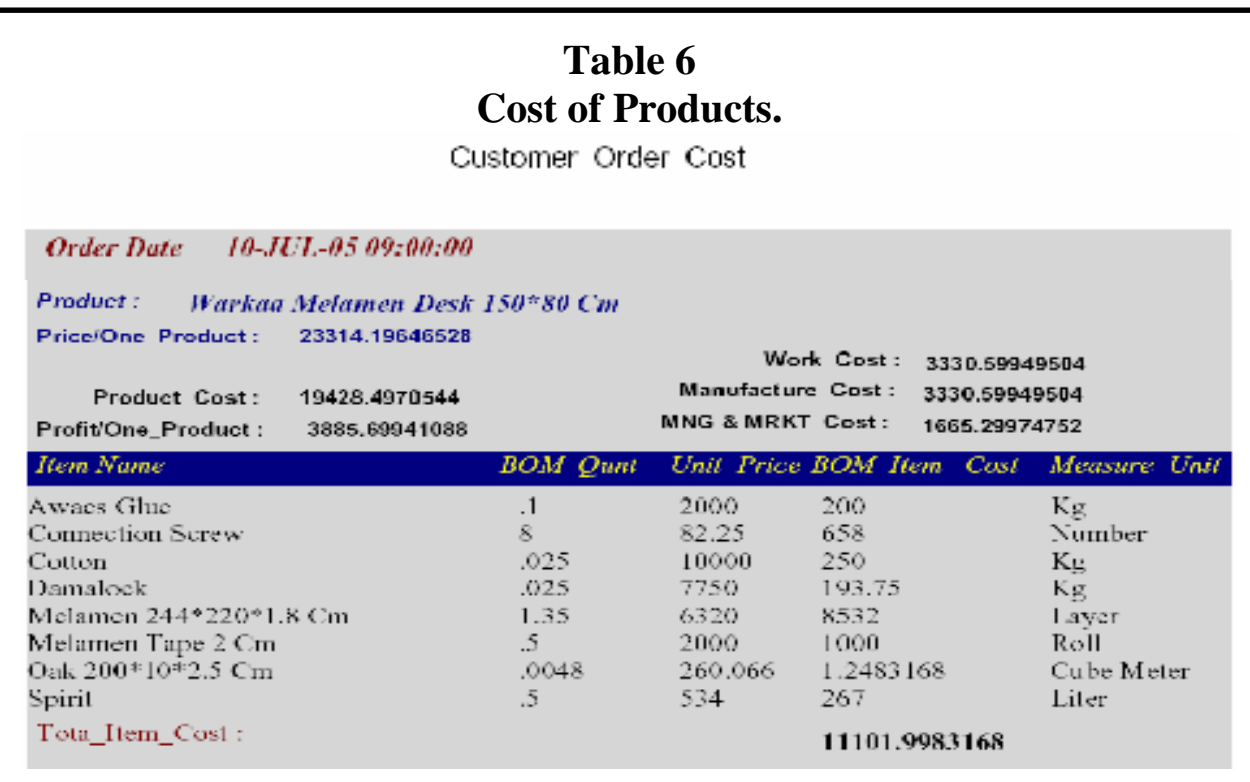




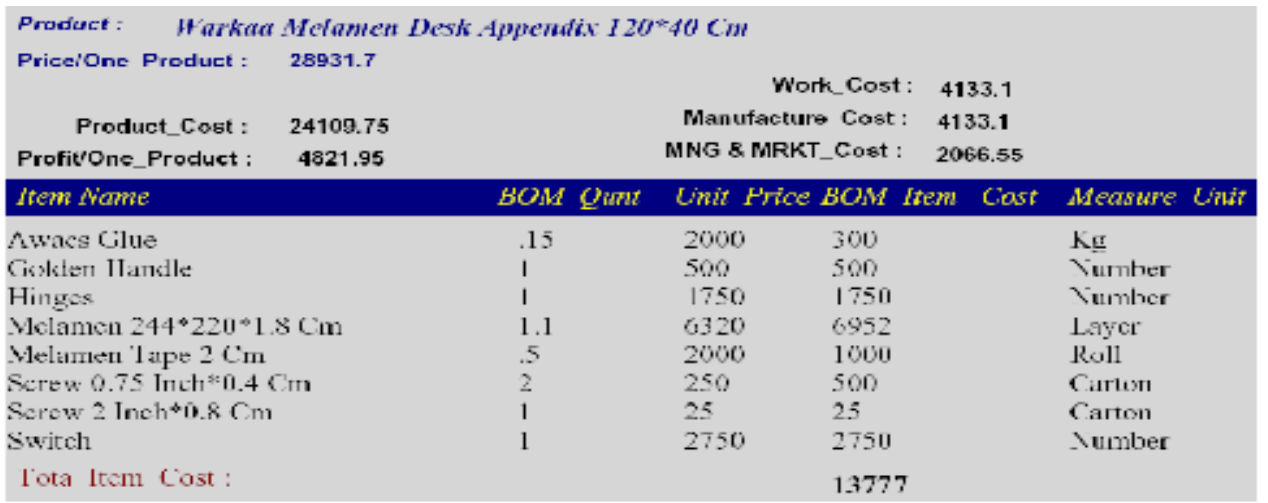

According to what has been mentioned above, table (7) illustrates the softwares execution results according to $\boldsymbol{A C T}$ function. The execution outlets a certain sequence for the jobs each time. According to this sequence the value of the fitness function is estimated. The best sequence for jobs executions has been selected according to the lowest value of fitness function of 10.143 as explained in the Table (7).

After calculating the order's lead time with the products involved, estimating the product's costs and consequently the order's total cost, the customer is informed with all this information as explained in the Table (8). As soon as the customer agrees on this, the Supervisor Agent will issue an order release for the product to be manufactured in the factory.

Table 7

The Optimal Job Sequence According to Average Completion Time Order Date [(1-1)7-105 (10);00;:00

Fitness Function : average completion time

\begin{tabular}{|c|c|c|}
\hline n_Time $\{$ A.C.T $)$ : & 10.143 & Sum_Tlow_Time(S.T.T): 71 \\
\hline Urilization ( 1$)$ : & 35.211 & Sun Prooss line $(S . P$ I' $)$ : \\
\hline 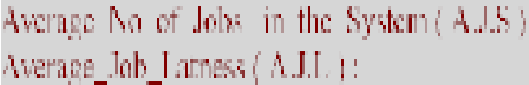 & $\begin{array}{l}2.84 \\
8.143\end{array}$ & Su _un_Job_attess (S.JiL) \\
\hline
\end{tabular}
Sinhs

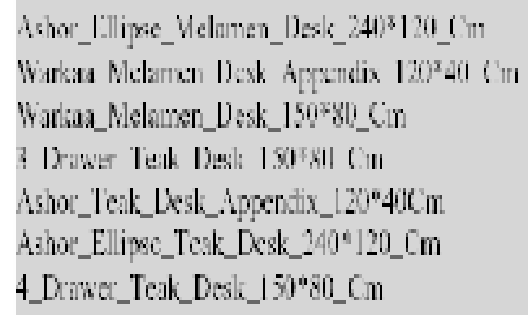


Table 8

Manufacturing Order's Price and Lead-time.

Product_Order (Price \& Lead_time)

10-JUL-0509:00:00

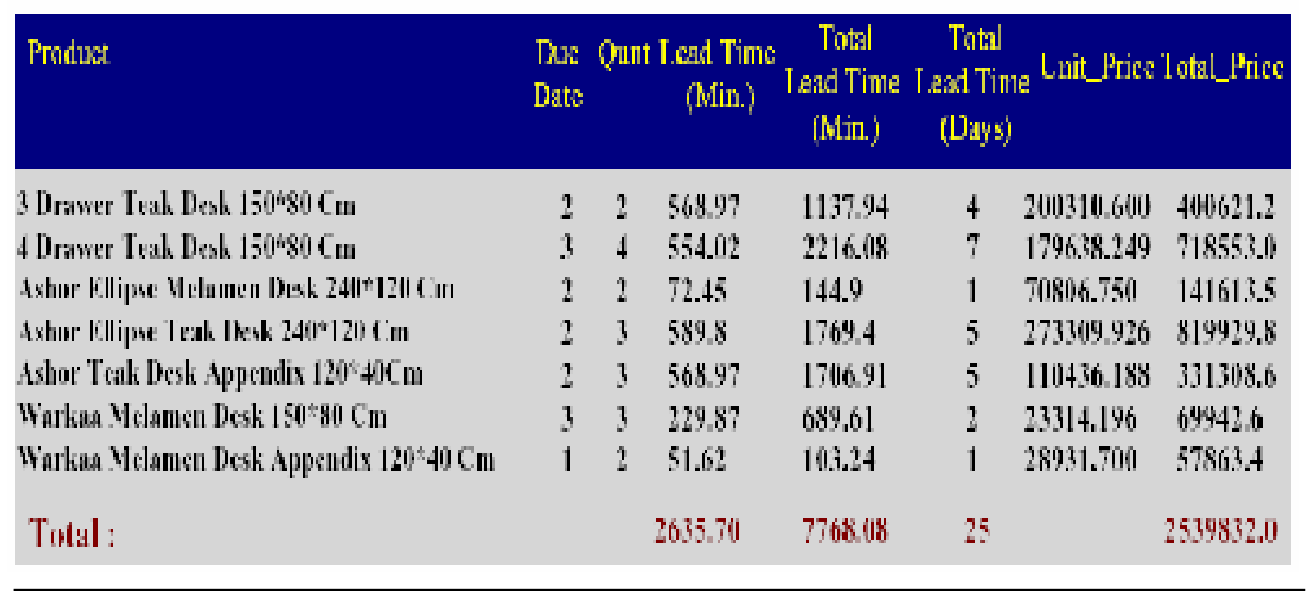

\section{Discussion and Analysis of the Application Results}

It has been shown so far that the basic idea of our system design and its application is to manufacture a product by the use of the computer, as procedures end as soon as commands are given to manufacture an order at the shop floor inside the factory. Accordingly, the application process represents assimilation to the actual manufacturing environment under study. As it has been explained before two orders which have, to some extent, some different features have been chosen. In order to explain the capabilities of the designed system at different stages of manufacturing, and within the framework of integration between (CAD/CAPP/HLMRP) and after all for the sake of implementing the philosophy of the virtual manufacturing, each order consists of a number of products. In the same way and concept the network, designed by the use of window server 2003 to the server and the window XP assigned to the clients, has a great role in achieving the concept of the VM. This has been demonstrated by the efficient flow of information and data throughout the manufacturing environment.

While the application has embodied the second objective of the thesis, it has, at the same time, provided integrated information to attain the other objectives.

\section{Conclusions}

1. The literature review of this research has revealed that the virtual manufacturing is based on Knowledge Engineering , manufacturing 
technology, system modeling technology and imitate reality technology that are supported by computer technology.

2. The resulting software will take manufacturing process plans as inputs and automatically generate a manufacturing schedule for a shop floor, a plant or an enterprise, with estimation of production costs and delivery options. This will allow the manufacturing enterprise to respond to customer requests quickly and therefore to win in the increasingly competitive markets.

3. The application results indicate that the VM philosophy has been performed efficiently by the use of the intelligent Multi-Agents which is managed and which attains integration by the server Agent that uses the available interfaces as Oracle language capabilities such as DDE, OLE and run-product. These capabilities have contributed in achieving quick response to the customers' orders.

4. The application results have confirmed that the use of Multi-Agent philosophy decreases the load on the server as far as each Agent represents a manufacturing cell. This means that the product manufacturing operations are distributed on the processing units .

5. The application and design operations have confirmed that the Oracle is distinguished by high efficiency as being easily compatible with the adopted software whether Oracle with AutoCAD or Oracle with $\mathrm{C}^{++}$. The Oracle is also distinguished by its ability to prepare a network Softwarely easily and make configuration on that net.

6. The practical part of the designed system has confirmed that the Agent based on manufacturing using Oracle in designing the database has achieved reality by capabilities such as triggers, privileges and role. This, undoubtedly, achieves speed, accuracy and security in information exchange.

7. The DDE and OLE interface tools are described to be contributing in making the designed system to have open system architecture which in its turn makes its suitable for the philosophy of the agile manufacturing.

8. The designed system is distinguished by having previously easy and rapid communication among the users. The actual dialogue among the users including the customer is considered to be one of the main goals of the VM system.

9. The Oracle possesses, through the enterprise manager console, the ability to give different privileges to every field within the database table.

\section{References}

1. Bohemia E., Working Collaboratively in Today Global Environment: A Global Product Development Course. Internet Engineering and Product Design Conference, 2004. 
2. Byrne, J., The virtual corporation: the company of the future will be the ultimate in adaptability, Business Week, 1993.

3. Chris M., Jimmie Browne, CADCAM principles, practice and manufacturing management, 2nd edition, Addison Wesley, 1998.

4. Christian, I. and others, Agile manufacturing transitional strategies, Proceedings of the Fourth SMESME International Conference, 2001.

5. Dai F., Gobel M.: Virtual prototyping-An approach using VR-techniques, in proceedings of the ASME international computers in engineering conference, September 11-14, Minneapolis, Minnesota, USA, 1994.

6. Herrera F., M. Lozano, and J. L. Verdegay, 'Tuning Fuzzy Logic Controllers by Genetic Algorithms', International Journal of Approximate Reasoning, 1995.

7. Highsmith, J., What is agile software development? The Journal of Defense Software Engineering, October 2002

8. Hondroudakis A., Malard,J.,Wilson, 'G.V.A An Introduction to Genetic Algorithm Using RPL2 EPCC TRACS', Student note 1995.

9. Kress H., Kiolein I., Rix J.: CAD data preparation in a virtual prototyping environment, in proceedings of the IFIP conference on life-cycle modeling for innovative products and processes, 1995.

10. Leo J. De Vin, Amos H.C., Jan Oscarsson, ,Simulation Based Decision Support for Manufacturing System Life Cycle Management, Published in Journal of Advanced Manufacturing Systems, Volume 3 Number 2, December 2004.

11. NIIIP, National Industrial Information Infrastructure Protocols Project, 2005, [www.niiip.org/public/home.nsf/vPubDocsByDocID/NIIP-

12. Pinedo, M., Scheduling Theory: Algorithms and Systems, Prentice Hall, Second Edition, 2002.

13.Putnik, E.D., Cunha, M.M., Sousa, R. and Avila, P., Virtual enterprise integration: technological and organization perspectives, Idea Group Publishing, 2005.

14. Roller D., Brunet P., CAD systems development tools and methods, springer, 1997.

15. Satish Jain,'Informatics practices', BPB publications, Vol. 1, 2004.

16. Schroeder K., Kress H.: Distributed conferencing tools for product design, in proceedings of the IFIP-workshop on interfaces in industrial systems for production and engineering, march 15-17, 1993, Elsevier publishers.

17. SQL Anywhere 10 Introduction, Inc. Portions Sybase, 2006, http://www.ianywhere.com/trademarks.

18. Takeshi Y., Ryohei N., 'A genetic algorithm with multi-step crossover for job-shop scheduling problems', International conference on genetic algorithms in engineering systems: innovations and applications, 1995.

19. TAMER F. ABDELMAGUID, MAGED M. DESSOUKY, 'A genetic algorithm approach to the integrated inventory distribution problem', 2004, http://wwwrcf.usc.edu/ maged/publications/GAinventoryrouting.pdf, Email: maged@usc.edu

20. Weiming Shen, Sherman Lang, Vibhor Gupta, Peter Orban, Lihui Wang, Qi Hao, 'Application of Intelligent Systems Technologies to Advanced Manufacturing', By Integrated Manufacturing Technologies Institute (IMTI), National Research Council Canada, 2005. 